\title{
Characteristics of Amine-Ended and Thiol-Ended Alkane Single-Molecule Junctions Revealed by Inelastic Electron Tunneling Spectroscopy
}

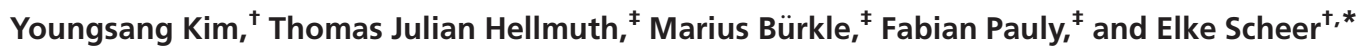 \\ ${ }^{\dagger}$ Department of Physics, University of Konstanz, 78457 Konstanz, Germany, and ${ }^{\ddagger}$ Institut für Theoretische Festkörperphysik, Karlsruhe Institute of Technology, \\ 76131 Karlsruhe, Germany
}

$\int$ ingle-molecule contacts are extensively studied using various methods because of their promising functionality as active electronic components and their interesting charge transport characteristics. $^{1-6}$ The role of the specific electrode material and the anchoring groups of the contacted molecules received special attention in recent years, because they turn out to be crucial for charge transport through molecular junctions, especially single-molecule junctions. $^{7-11}$ The choice of the anchoring group can tune the charge transport behavior because the binding energy and preferred binding site of the anchoring group to the metallic electrode determine the contact geometry. The resulting alignment of molecular states with respect to the Fermi energy plays a dominant role in the conductance of molecular junctions. ${ }^{12-16}$ Among several different anchoring groups of molecules, the amine $\left(\mathrm{NH}_{2}\right)$ end group has been suggested as a promising building block due to its selective binding to low-coordinated Au surface atoms, resulting in a narrow distribution of molecular conductance values. ${ }^{12-17}$ However, depending on temperature, speed of contact formation, and further external parameters, also wider conductance distributions of the amine-ended molecules have been reported. ${ }^{18}$ To thoroughly understand the transport mechanism in the hybrid metalmolecule structures, a more sophisticated measurement is required, and inelastic electron tunneling spectroscopy (IETS) has been introduced due to its high sensitivity to the molecular configuration and contact geometry. ${ }^{6,19-21}$

In this article, the charge transport through single 1,8-octanedithiol (ODT, HS- $\left[\mathrm{CH}_{2}\right]_{8}-\mathrm{SH}$ )
ABSTRACT A combined experimental and theoretical analysis of the charge transport through single-molecule junctions is performed to define the influence of molecular end groups for increasing electrode separation. For both amine-ended and thiol-ended octanes contacted to gold electrodes, we study signatures of chain formation by analyzing kinks in conductance traces, the junction length, and inelastic electron tunneling spectroscopy. The results show that for amine-ended molecular junctions no atomic chains are pulled under stretching, whereas the Au electrodes strongly deform for thiol-ended molecular junctions. This advanced approach hence provides unambiguous evidence that the amine anchors bind only weakly to Au.

KEYWORDS: molecular electronics · conductance measurement $\cdot$ inelastic electron tunneling spectroscopy · single-molecule junction · anchoring groups $\cdot$ density functional theory

and 1,8-octanediamine (ODA, $\mathrm{H}_{2} \mathrm{~N}-\left[\mathrm{CH}_{2}\right]_{8}$ $\mathrm{NH}_{2}$ ) molecules contacted to Au electrodes using mechanically controllable break-junctions (MCBJ) is measured at $4.2 \mathrm{~K}$. A statistical analysis of the conduction properties as a function of junction length ("opening conductance") is performed, supplemented by theory. We focus particularly on potential signals of chain formation upon junction elongation. Peaks in the IETS spectra are assigned by combined $a b$ initio electronic structure and transport calculations, taking into account the inelastic effects due to the electron-vibration coupling.

\section{RESULTS AND DISCUSSION}

Linear Conductance. The ODT and ODA molecules are contacted to the Au electrodes by the MCBJ technique as illustrated in Figure 1a. To investigate the charge transport through the single-molecule junctions, the samples are mounted in the custommade cryostat equipped with the MCBJ system, and then all the transport measurements are performed at $4.2 \mathrm{~K}$ (see Methods). In order to characterize the conductance of @2011 American Chemical Society 
A

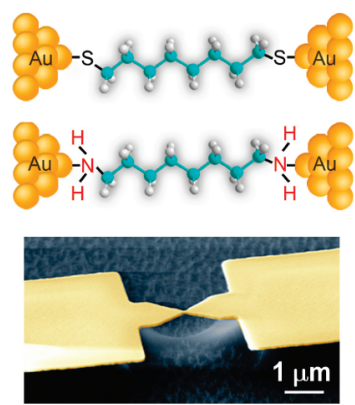

C

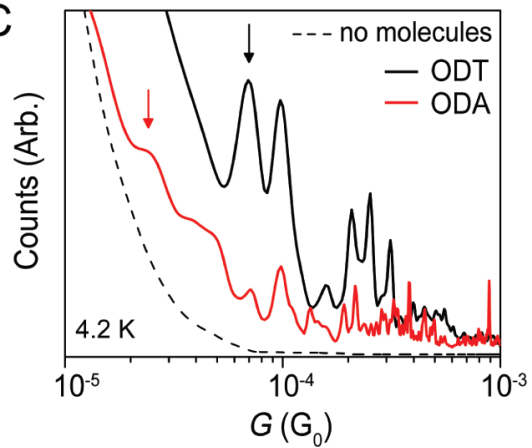

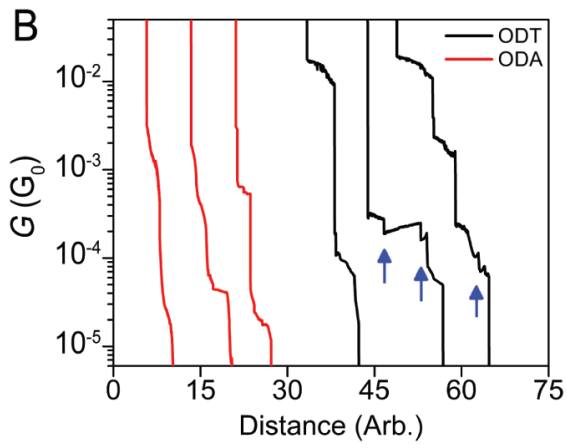

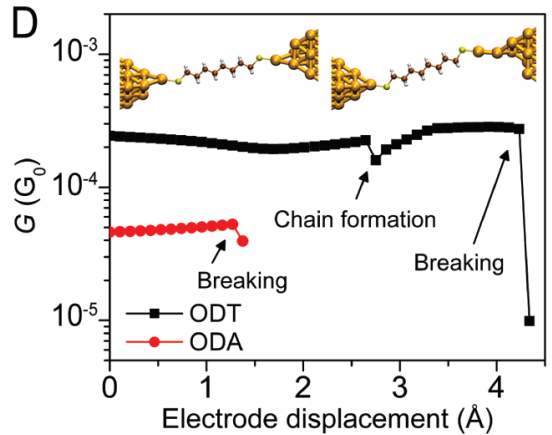

Figure 1. (a) Illustrations of ODT and ODA single-molecule junctions and scanning electron microscopy image of a MCBJ sample. (b) The conductance traces of ODT (black) and ODA (red) molecular junctions are obtained by applying $70 \mathrm{mV}$ of dc voltage and are shown for the opening process. The arrow marks a representative kink or dip at which the slope of the conductance plateau upon stretching changes sign. (c) Conductance histograms of ODT (black) and ODA (red) molecular junctions. Both histograms are collected by repeating the opening and closing process 300 times and are displayed for a bin size of $3 \times 10^{-6} G_{0}$. The dashed line presents the background conductance measured under vacuum (no molecules). The arrows indicate the lowest conductance peaks of the histograms. Junctions with these conductance values are used for the IETS measurements. (d) Calculated conductance of ODT and ODA junctions as a function of the electrode displacement. The kink in the ODT trace around $2.8 \AA$ indicates the formation of an Au chain. The simulated atomic arrangements, shortly before and after the chain forms, are presented in the inset.

the metal-molecule junctions, we repeatedly opened and closed them and recorded conductance-distance traces as displayed in Figure $1 \mathrm{~b}$. The conductance histograms measured with molecules (ODT and ODA) and without molecules are presented in Figure 1c. The appearance of multiple conductance peaks as shown in the histogram of Figure 1c was observed in different experimental setups (e.g., with scanning tunneling microscopes or break-junctions) previously. ${ }^{8,16,18,22,23}$ This observation can be attributed to the formation of contact geometries with different configurations of the molecule, of the electrodes, or at the molecule-electrode interface ${ }^{8,9,22}$ and to junctions containing multiple molecules that contribute to the current. ${ }^{8}$ In the present study we restrict ourselves to the investigation of junctions with the lowest stable conductance, as indicated by the position of the lowest conductance peak. They are supposed to correspond to single-molecule junctions. The minimum conductance values are shown by arrows in Figure 1c. By fitting a Lorentzian function to the lowest peak in the histogram, we obtain $(7.0 \pm 1.2) \times 10^{-5} G_{0}$ for ODT and $(3.0 \pm 0.5) \times 10^{-5} G_{0}$ for ODA, where the error is determined by the halfwidth at half-maximum (hwhm) and $G_{0}=2 e^{2} / h$ is the quantum of conductance.
For a better understanding, we performed simulations of the stretching of ODT and ODA single-molecule junctions using a DFT-based scheme (see Methods). ${ }^{24}$ Besides the equilibrium structures at the different elongation stages, we determined the conductance and find it to range between 1 and $3 \times$ $10^{-4} G_{0}$ for ODT contacts and between 4 and $6 \times$ $10^{-5} G_{0}$ for ODA, as shown in Figure $1 \mathrm{~d}$ (see also the Supporting Information (SI)). ${ }^{25}$ Compared to the experiment, the conductance is overestimated in both cases by a factor of around 3. This may be related to uncertainties in the description of the alignment of the molecular states and the metal Fermi energy, as well as the band gap problem of DFT. ${ }^{26-29}$ However, the deviations appear acceptable considering the lack of knowledge with respect to the precise contact geometry. The reduced conductance, by a factor of around 5, for similar junction geometries of ODA as compared to ODT is in qualitative agreement with the experimental observations and previous reports in the literature. $^{15,18}$ We find that the transport for both ODT and ODA is dominated by electronic states with a high weight on the anchoring groups of the molecules and is slightly more off-resonant for ODA than ODT (see SI). 
Inelastic Electron Tunneling Spectroscopy. To date, several IETS studies have been performed using thiolended molecules. ${ }^{6,19,20}$ However, IETS measurements on amine-ended molecules have not been reported so far in spite of their promising transport properties. The vibrational modes of the molecules are detected by their effect on the current through the molecular tunnel barrier. In this process the excitation of a molecular vibration by the charge carriers gives rise to a peak in the $d^{2} I / d V^{2}$ in the typical off-resonant situation. ${ }^{6,19,21}$

We measure an antisymmetric $I-V$ and IETS of both $\mathrm{Au}-\mathrm{ODT}-\mathrm{Au}$ and Au-ODA-Au junctions, which indicates that the molecules are symmetrically coupled to both electrodes. ${ }^{19-21}$ Furthermore, we find a temperature-independent $I-V$ for studies between 4.2 and $42 \mathrm{~K}$, signaling tunneling transport. Both of these results are shown in the $\mathrm{SI}$.

For the IETS measurement, we normalize the second derivative of the $I-V$ curves $\left(d^{2} I / d V^{2}\right)$ with the differential conductance ( $\mathrm{d} / \mathrm{d} V$ ) in order to compensate for the conductance change due to distance changes of the electrodes. Hence, the IETS amplitude is defined as $\left(\mathrm{d}^{2} I / \mathrm{d} V^{2}\right) /(\mathrm{d} l / \mathrm{d} V) .{ }^{19,30}$ Both derivatives are measured as the first and second harmonic signals, using lock-in techniques. We applied a dc bias to the samples added to an ac modulation of $6 \mathrm{mV}$ (rootmean-square) at a frequency of $317 \mathrm{~Hz}$ (see Methods). Once a stable junction has been established, we were able to sweep the bias voltage several times without significant change of the IETS. This allows us to perform repeated IETS measurements of the same junction. With changing electrode distance the IETS spectra vary; that is, peaks vary in height and positions. The positions may shift by some $10 \mathrm{mV}$ due to the changes in the junction geometry (see discussion below).

Figure $2 a$ and $b$ shows measured IETS spectra of $\mathrm{Au}-\mathrm{ODT}-\mathrm{Au}$ and $\mathrm{Au}-\mathrm{ODA}-\mathrm{Au}$ junctions, respectively. In the low-energy regime of the IETS spectra, i.e., below $200 \mathrm{mV}$ (which is regarded as the "fingerprint regime" of a molecular junction because the fundamental vibrational modes typically fall in this energy range), we observe prominent molecular vibrational peaks for both ODT and ODA molecular junctions. Due to the many modes below $25 \mathrm{mV}$, these energies are excluded from the discussion in this subsection, but we will come back to them when we consider Figure 4 .

We characterize the vibrational modes both by performing DFT calculations of the eigenmodes of the molecular junctions and by computing IETS spectra by means of a lowest order expansion in the electronvibration coupling (see Methods). In this way, we determine peak positions, peak heights, and the evolution of vibrational energies during the stretching of the molecular contacts.

Following the experiments, we broaden our theoretical IETS spectra by an ac voltage of $6 \mathrm{mV}$ (root-meansquare). ${ }^{31,32}$ We show them as the dashed lines beside
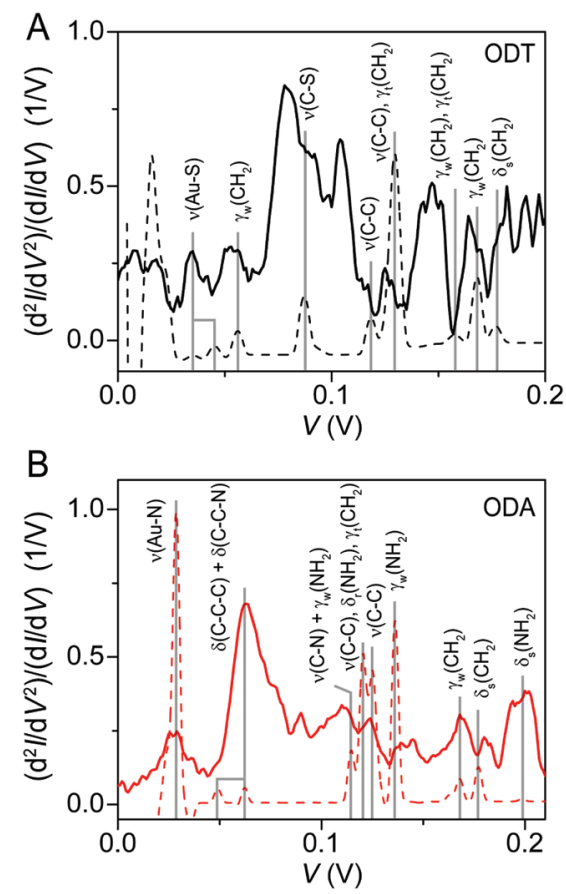

Figure 2. Comparison of experimental (solid lines) and theoretical (dashed lines) IETS spectra of (a) ODT and (b) ODA single-molecule junctions. Experimental results are obtained from the lock-in second-harmonic signals at $4.2 \mathrm{~K}$. The vertical lines show the peak positions in the theoretically obtained spectra, and modes of the same character are summarized by horizontal lines. The character of the modes is specified by the symbols explained in the text. The separation by a comma means that the peak is due to several modes, while " + " is used to indicate the mixed character of a mode.

the experimental spectra in Figure 2. The main character of those modes is indicated, which are responsible for the peaks in the calculated spectra. Since the theoretical IETS spectra have been calculated close to our starting geometries (see the geometries with an elongation of $0.00 \AA$ in the $\mathrm{SI}$ ), we compare them to experimental spectra taken from the middle part of stretching experiments, because in this situation a straight, but not strongly elongated molecular junction can be assumed. Those modes identified by the comparison between theory and experiment are summarized in Table 1.

According to our analysis, the peak at around $35 \mathrm{mV}$ in Figure 2a results from the $v(\mathrm{Au}-\mathrm{S})$ stretching mode and those at $29 \mathrm{mV}$ in Figure $2 \mathrm{~b}$ from the $v(\mathrm{Au}-\mathrm{N})$ stretching mode. They signal that both amine and thiol end groups are robustly bonded to the Au atomic electrodes. Further main peaks in the experimental IETS at around 53, 78, 125, 164, and $182 \mathrm{mV}$ for the AuODT-Au junction are attributed to $\gamma_{\mathrm{w}}\left(\mathrm{CH}_{2}\right)$ wagging, $v(\mathrm{C}-\mathrm{S})$ stretching, $v(\mathrm{C}-\mathrm{C})$ stretching and $\gamma_{\mathrm{t}}\left(\mathrm{CH}_{2}\right)$ twisting, $\gamma_{w}\left(\mathrm{CH}_{2}\right)$ wagging, and $\delta_{s}\left(\mathrm{CH}_{2}\right)$ scissoring, respectively, in accordance with previous studies. ${ }^{6,10,19,20,33-39}$ For the $\mathrm{Au}-\mathrm{ODA}-\mathrm{Au}$ junction we assign the main peaks at $63,110,124,139,168,180$, and $200 \mathrm{mV}$ to combined $\delta(\mathrm{C}-\mathrm{C}-\mathrm{C})$ and $\delta(\mathrm{C}-\mathrm{C}-\mathrm{N})$ bending, combined $\nu(\mathrm{C}-\mathrm{N})$ stretching and $\gamma_{\mathrm{w}}\left(\mathrm{NH}_{2}\right)$ wagging, $v(\mathrm{C}-\mathrm{C})$ stretching, 
TABLE 1. Summary of the Vibrational Mode Assignment in the IETS Spectra for ODT and ODA Molecular Junctions, Shown in Figure $2^{a}$

\begin{tabular}{|c|c|c|c|c|}
\hline \multirow[b]{2}{*}{ molecule } & \multirow[b]{2}{*}{ modes $^{b}$} & \multirow[b]{2}{*}{ description $^{b}$} & \multicolumn{2}{|c|}{ peak position (mV) } \\
\hline & & & experiment & theory \\
\hline \multirow[t]{8}{*}{ ODT } & $v(\mathrm{Au}-\mathrm{S})$ & $\mathrm{Au}-\mathrm{S}$ stretching & 35 & 35,45 \\
\hline & $\gamma_{\mathrm{w}}\left(\mathrm{CH}_{2}\right)$ & $\mathrm{CH}_{2}$ wagging & 53 & 56 \\
\hline & $v(C-S)$ & C-S stretching & 78 & 86 \\
\hline & $v(\mathrm{C}-\mathrm{C})$ & C $-C$ stretching & & 118 \\
\hline & $v(\mathrm{C}-\mathrm{C}), \gamma_{\mathrm{t}}\left(\mathrm{CH}_{2}\right)$ & $\mathrm{C}-\mathrm{C}$ stretching, $\mathrm{CH}_{2}$ twisting & 125 & 130 \\
\hline & $\gamma_{\mathrm{w}}\left(\mathrm{CH}_{2}\right), \gamma_{\mathrm{t}}\left(\mathrm{CH}_{2}\right)$ & $\mathrm{CH}_{2}$ wagging, $\mathrm{CH}_{2}$ twisting & & 157 \\
\hline & $\gamma_{w}\left(\mathrm{CH}_{2}\right)$ & $\mathrm{CH}_{2}$ wagging & 164 & 168 \\
\hline & $\delta_{s}\left(\mathrm{CH}_{2}\right)$ & $\mathrm{CH}_{2}$ scissoring & 182 & 178 \\
\hline \multirow[t]{9}{*}{ ODA } & $v(\mathrm{Au}-\mathrm{N})$ & $\mathrm{Au}-\mathrm{N}$ stretching & 29 & 29 \\
\hline & $\delta(\mathrm{C}-\mathrm{C}-\mathrm{C})+\delta(\mathrm{C}-\mathrm{C}-\mathrm{N})$ & $\mathrm{C}-\mathrm{C}-\mathrm{C}$ bending with $\mathrm{C}-\mathrm{C}-\mathrm{N}$ bending & 63 & 50,64 \\
\hline & $v(\mathrm{C}-\mathrm{N})+\gamma_{\mathrm{w}}\left(\mathrm{NH}_{2}\right)$ & $\mathrm{C}-\mathrm{N}$ stretching with $\mathrm{NH}_{2}$ wagging & 110 & 115 \\
\hline & $v(\mathrm{C}-\mathrm{C}), \delta_{\mathrm{r}}\left(\mathrm{NH}_{2}\right), \gamma_{\mathrm{t}}\left(\mathrm{CH}_{2}\right)$ & $\mathrm{C}-\mathrm{C}$ stretching, $\mathrm{NH}_{2}$ rocking, $\mathrm{CH}_{2}$ twisting & & 121 \\
\hline & $v(\mathrm{C}-\mathrm{C})$ & $C-C$ stretching & 124 & 126 \\
\hline & $\gamma_{\mathrm{w}}\left(\mathrm{NH}_{2}\right)$ & $\mathrm{NH}_{2}$ wagging & 139 & 137 \\
\hline & $\gamma_{\mathrm{w}}\left(\mathrm{CH}_{2}\right)$ & $\mathrm{CH}_{2}$ wagging & 168 & 169 \\
\hline & $\delta_{s}\left(\mathrm{CH}_{2}\right)$ & $\mathrm{CH}_{2}$ scissoring & 180 & 178 \\
\hline & $\delta_{s}\left(\mathrm{NH}_{2}\right)$ & $\mathrm{NH}_{2}$ scissoring & 200 & 199 \\
\hline
\end{tabular}

${ }^{a}$ Peak positions in the spectra are identified by our IETS calculations and previous IETS experiments and calculations for 0 DT. ${ }^{6,10,19,20,33-39 b}$ When we separate modes by a comma, there are several contributing to the same peak. When we use " + " or "with", a single mode has a mixed character.

A

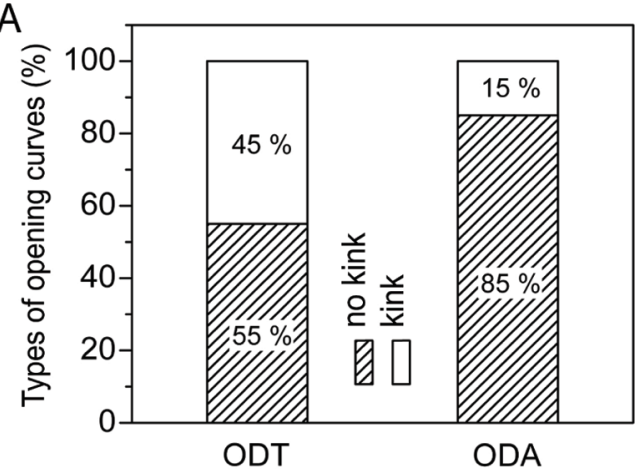

B

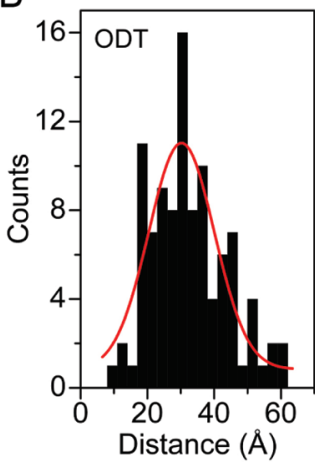

$\gamma_{\mathrm{w}}\left(\mathrm{NH}_{2}\right)$ wagging, $\gamma_{\mathrm{w}}\left(\mathrm{CH}_{2}\right)$ wagging, $\delta_{\mathrm{s}}\left(\mathrm{CH}_{2}\right)$ scissoring, and $\delta_{s}\left(\mathrm{NH}_{2}\right)$ scissoring, respectively.

As expected, the higher energy modes above $100 \mathrm{meV}$, affecting mainly the $\mathrm{C}$ and $\mathrm{H}$ atoms of the molecule, appear at similar energies for ODA and ODT, however with different amplitudes. Modes including motions of the $\mathrm{NH}_{2}$ groups appear in a broad energy range. We observe that some modes appearing in the experimental spectra are absent in the theoretical ones and vice versa. This discrepancy is due to the fact that only single spectra are compared, likely corresponding to different contact configurations. Choosing the peak at $104 \mathrm{mV}$ in the spectrum of ODT as an example, it has been shown ${ }^{38}$ that the $\delta_{r}\left(\mathrm{CH}_{2}\right)$ rocking modes, to which we ascribe the peak, may be excited only in certain configurations, which lift particular symmetries. Other prominent features, not explained by our calculations, could be due to combined $\gamma_{w}\left(\mathrm{CH}_{2}\right)$ wagging and $\gamma_{\mathrm{t}}\left(\mathrm{CH}_{2}\right)$ twisting modes at $147 \mathrm{meV}$ for ODT and $\delta_{\mathrm{r}}\left(\mathrm{CH}_{2}\right)$ rocking modes at $90 \mathrm{meV}$ for ODA.

In summary, the detailed comparison of theoretical and experimental IETS spectra reveals the complex interplay of molecular conformation and inelastic transport. Changing the anchoring group has an important influence on the vibrational spectrum and the possibility to excite modes electrically. In the following we shall concentrate on metal-anchor group vibrations, and also the low-energy Au vibrational modes are discussed in this context below.

The Gold-Molecule Interface. According to our theoretical investigations, the binding energy of the $\mathrm{Au}-\mathrm{N}$ 
bond $(0.92 \mathrm{eV})$ is significantly weaker than those of the $\mathrm{Au}-\mathrm{S}$ bond $(1.76 \mathrm{eV})$ for the binding in top position (see $\mathrm{SI}){ }^{12,40}$ Indeed, in our simulations we find that this difference results in the pulling of Au chains for ODT, ${ }^{10,41}$ while they are absent for ODA (see Figure 1d and the SI).

Figure $1 \mathrm{~d}$ shows an example of a calculated opening trace revealing a kink between two rather flat
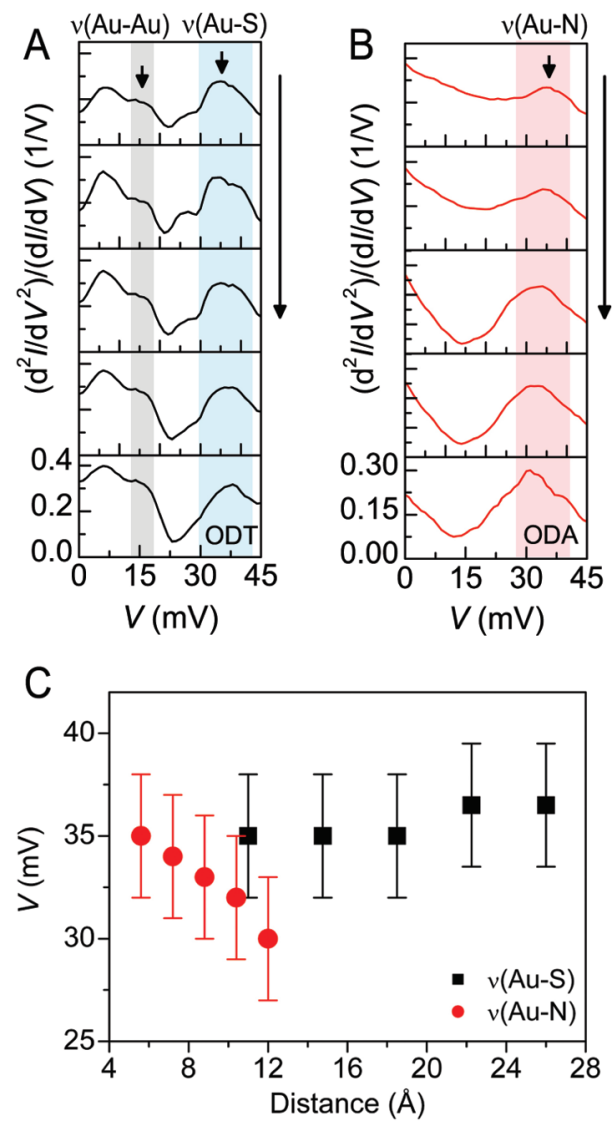

Figure 4. $(a, b)$ The evolution of the IETS spectra, obtained at the lowest conductance plateaus for dc bias voltages up to $45 \mathrm{mV}$, is shown for the stretching (in the order of the arrow) of a single-junction realization for ODT and ODA, respectively. $y$-axes are of the same scale in each panel. (c) Evolution of the $v(\mathbf{A u}-\mathbf{S})$ and $v(\mathbf{A u}-\mathbf{N})$ modes as extracted from panels $a$ and $b$. The error bars are defined by the applied ac modulation voltage of $6 \mathrm{mV}$.

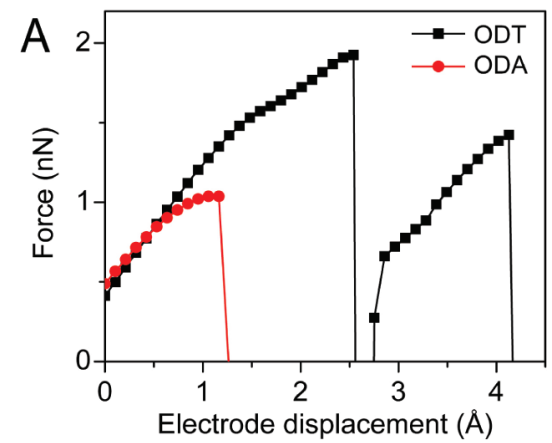

plateau regions at the moment when the Au chain starts to be formed. In the experiments, however, the conductance plateaus usually exhibit a negative slope throughout the whole stretching process. Therefore, we identify a kink by a change of the conductance-distance slope from negative to positive, as shown by the arrow in Figure 1b. We use their appearance in the opening conductance as an indication of chain formation or, more generally, a mechanical deformation of the Au electrodes.

As displayed in Figure $3 a$, we find that $45 \%$ of the traces show kinks for the ODT junctions, whereas they occur in only $15 \%$ of the ODA junctions. Hence, the low percentage of the kinks in ODA junctions signals that the amine anchors do not cause strong mechanical deformations of the Au electrodes during stretching. Moreover, the average plateau lengths upon stretching of Au-ODT-Au junctions ( $30 \pm 11 \AA$ ) are significantly larger than those of $\mathrm{Au}-\mathrm{ODA}-\mathrm{Au}$ junctions (11 $\pm 3 \AA$ ), as shown in Figure $3 b$,c. Here, the average length is specified by the maximum of the Gaussian fit to the measured data, while the error is determined by the hwhm.

In the experiment, however, the apparent plateau lengths are much larger than those in the simulations. We observe from Figure $1 \mathrm{~d}$ that the electrode displacement of the ODT junction can be changed by $\sim 4.4 \AA$ before rupture, while this displacement for ODA of $\sim 1.4 \AA$ is clearly smaller. Although this agrees qualitatively with the differences in average single-molecule plateau lengths of Figure 3, the discrepancy between experiment and theory amounts to a factor of $\sim 10$. We attribute this to the fact that we start in our calculations with rather straight molecules bonded to the ends of Au tips (see also Figures S4 and S5 of the SI). In the experiments, the molecules can be twisted and stretched upon elongation, or their anchoring group may "slide" along the metallic electrodes on both sides toward the tip ends. This explains why the plateau-length histogram for ODA is peaked at around $12 \AA$, the length of the isolated ODA molecule ( $\mathrm{N}-\mathrm{N}$ distance). For ODT deformations of the metal electrode can arise in addition, which we cannot account for entirely in the calculations due to the limited amount of flexible atoms in the $\mathrm{Au}$ electrodes.

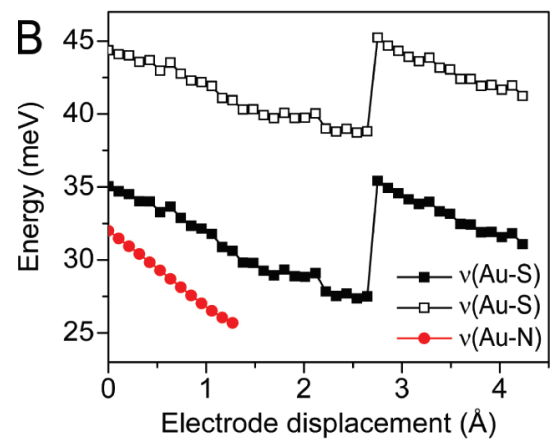

Figure 5. Theory results for (a) the tension force and (b) the vibrational energies of gold-anchor group stretching modes as a function of the electrode displacement for ODT and ODA molecular contacts. The $v(\mathbf{A u}-\mathbf{S})$ and $v(\mathbf{A u}-\mathbf{N})$ modes shown are those causing the peaks in the calculated IETS spectra in Figure 2. 
Nevertheless, the experimental and theoretical results consistently suggest that strong mechanical deformations of the Au electrodes are involved for ODT, possibly including the formation of Au chains, while such deformations are essentially absent for ODA.

The different behavior of thiol-ended and amineended molecular junctions is further elucidated, when we compare the $v(\mathrm{Au}-\mathrm{S})$ and $v(\mathrm{Au}-\mathrm{N})$ vibrational modes in the IETS spectra for increasing electrode separation in the lowest conductance regime. In Figure $4 a$, b the IETS spectra below $45 \mathrm{mV}$ are presented for increasing stretching distance in the order from top to bottom. In Figure $4 \mathrm{a}$ the $v(\mathrm{Au}-\mathrm{S})$ mode is very stable, and no significant change of vibrational energy during the stretching of the junction can be noticed. In contrast, in the Au-ODA-Au junction of Figure $4 \mathrm{~b}$, the energy of the $v(\mathrm{Au}-\mathrm{N})$ mode is red-shifted by more than $5 \mathrm{mV}$ with increasing electrode separation. Figure $4 \mathrm{c}$ summarizes the evolution of the $v(\mathrm{Au}-\mathrm{S})$ and $v(\mathrm{Au}-\mathrm{N})$ vibrational peaks under stretching, showing clearly the red-shift of the $v(\mathrm{Au}-\mathrm{N})$ mode. This behavior is consistent with an increase of the $\mathrm{Au}-\mathrm{N}$ bond length due to its weak bond strength, going along with the reduction of force constants. These features were obtained in all continuous IETS measurements during the opening of the ODA and ODT contacts (see SI).

Interestingly, we observe an increase of IETS intensity at about $15 \mathrm{mV}$ as the electrode separation increases for ODT junctions in Figure 4a. On the basis of our DFT calculations (see the $\mathrm{SI}$ ) and in agreement with previous reports, ${ }^{42}$ we attribute this to an Au longitudinal vibrational mode $(v(\mathrm{Au}-\mathrm{Au}))$, which indicates the formation of a one-dimensional atomic chain. ${ }^{43}$ Moreover, we do not detect the $v(\mathrm{Au}-\mathrm{Au})$ signal around $15 \mathrm{mV}$ in ODA molecular junctions (see Figure $4 b$ ). This feature underlines that $A u$ atoms in ODA molecular junctions do not form one-dimensional chains upon stretching. However, we note that we observed zero-bias anomalies (ZBA) below $15 \mathrm{meV}$ instead of the peaks in the example shown, the origin of which is not well-understood so far. ${ }^{19,36}$

In Figure 5 a we show the calculated evolution of the tension force during the stretching process, obtained as the derivative of the total energy with respect to the displacement distance. Elastic and plastic stages can be distinguished, where forces increase roughly linearly during the elastic stages. Finally, the stress is released abruptly in plastic stages by mechanical deformation of the gold electrodes or bond rupture. We note that for the ODT contact that breaks at an $\mathrm{Au}-\mathrm{Au}$ bond, we obtain a breaking force of $1.5 \mathrm{nN}$, which is in good agreement with breaking forces measured for atomic chain geometries of Au atomic contacts. ${ }^{44}$ In contrast, the breaking force for ODA contacts of $1 \mathrm{nN}$ is lower. In Figure $5 \mathrm{~b}$ we plot the evolution of the energy of the $v(\mathrm{Au}-\mathrm{S})$ and $v(\mathrm{Au}-\mathrm{N})$ vibrations for ODT and ODA junctions, which lead to the peaks in the computed IETS spectra in Figure 2. We find a substantial red shift of the $v(\mathrm{Au}-\mathrm{S})$ and $v(\mathrm{Au}-\mathrm{N})$ vibrational energies upon stretching for both kinds of junctions. The $v(\mathrm{Au}-\mathrm{N})$ mode shows a behavior in good agreement with the experiment. In contrast, the decreasing frequency of the $v(\mathrm{Au}-\mathrm{S})$ modes during the elastic stages does not comply with the experimental observations. However, we expect that the calculations underestimate the deformability of the Au electrodes due to the limited reservoir of mobile Au atoms assumed for practical reasons. Indeed, when stress is released by pulling gold atoms out of the electrodes in the plastic stages, we observe a "revival", i.e., an increase, of the $v(\mathrm{Au}-\mathrm{S})$ vibrational frequencies. The experiments hence suggest that the force constants of the $\mathrm{Au}-\mathrm{S}$ stretching modes stay effectively constant on the experimental time scales due to the mechanical deformations of the metal electrodes. Overall, this comparison of $v(\mathrm{Au}-\mathrm{S})$ and $v(\mathrm{Au}-\mathrm{N})$ modes with the help of the experimental IETS measurements clearly demonstrates that the $\mathrm{Au}-\mathrm{N}$ bond is significantly weaker than the $\mathrm{Au}-\mathrm{S}$ bond.

\section{CONCLUSIONS}

We performed IETS measurements for both $\mathrm{Au}-$ ODT-Au and $\mathrm{Au}-\mathrm{ODA}-\mathrm{Au}$ single-molecule junctions at $4.2 \mathrm{~K}$ using the MCBJ technique. Our analysis was complemented by DFT-based calculations of junction properties under elongation, yielding information on stable contact geometries, elastic transport properties, and inelastic electron tunneling spectra by considering the electron-vibration coupling perturbatively to lowest order. We present for the first time inelastic transport data and the corresponding theoretical information for $\mathrm{Au}-\mathrm{ODA}-\mathrm{Au}$ junctions and find good agreement. Our results demonstrate that the amine-ended molecules bind only weakly to $\mathrm{Au}$, in contrast to the thiol-ended ones. In particular, the strong $\mathrm{Au}$-thiol bond causes major mechanical deformations of the Au electrodes, including the pulling of Au chains, while such deformations are largely absent for the quickly breaking Au-amine bonds. These findings can play an important role for the controlled design of future molecular electronic devices.

\section{METHODS}

Device Fabrication. The process of sample fabrication is as follows. The softly polished bronze wafer ( $200 \mu \mathrm{m}$ in thickness) is spin-coated with a layer of polyimide $(2 \mu \mathrm{m})$, which serves as an electrical insulator and a sacrificial layer in the subsequent etching process. On top of these prepared wafers, a double layer of electron-beam resists, MMA-MAA/PMMA, is deposited by spin-coating. The electron beam lithography process is performed on this electron beam resist coated bronze wafer $(4 \times$ $19 \mathrm{~mm}^{2}$ ). After developing, gold of about $80 \mathrm{~nm}$ thick is deposited using electron-beam evaporation at a pressure of 
about $10^{-8}$ mbar. Finally, in order to form a free-standing bridge, the polyimide layer is selectively etched away by about $700 \mathrm{~nm}$ employing $\mathrm{O}_{2}$ plasma in a vacuum chamber of a reactive ion etcher. ${ }^{45,46}$ The scanning electron microscopy image of the device fabricated in this way is shown in Figure 1a. For assembling the molecules on the samples, a dilute solution of molecules $(1 \mathrm{mM})$ in $10 \mathrm{~mL}$ of ethanol was prepared, and then the patterned substrates were immersed in the molecular solution for $5 \mathrm{~h}$. Each sample was then rinsed with ethanol and gently blown dry in a stream of nitrogen gas to remove noncovalently attached alkanedithiol or alkanediamine molecules from the metal surface. ${ }^{6,47}$

Low-Temperature Setup. Charge transport measurements through single molecules were carried out in a custom-designed cryogenic vacuum insert equipped with a MCBJ system for both Au-ODT-Au and Au-ODA-Au junctions. The devices are mounted into the break mechanism inside an inner vacuum chamber, which is evacuated and purged with He gas before being immersed into a liquid He dewar. The breaking mechanics is controlled by a dc motor with a gear box (reduction ratio of 1:1734) connected with a vacuum feed-through. In order to reduce the electronic noise level, all measurement lines inside the cryostat are carefully filtered using homemade coaxial cables and SMA connectors. Inside the inner vacuum chamber, the sample holder part is shielded again with a copper can to eliminate the influence of electromagnetic fields. ${ }^{46,48}$

Transport Measurement. The conductance measurements of opening and closing were performed by a subfemtoamp source-meter (Keithley 6430) operating with an automatic variable gain preamplifier. In order to measure the $I-V$ curves, we used a programmable dc source (Yokogawa 7651) and a low-noise current amplifier (Ithaco 1211) followed by a digital multimeter (Agilent 34401A). All grounds of the system were carefully designed to avoid ground-loops and electrical noise. All data were collected by Labview software through GPIB cables. The differential conductance $(\mathrm{d} / / \mathrm{d} V)$ and its derivative $\left(\mathrm{d}^{2} / / \mathrm{d} V^{2}\right)$ were measured simultaneously as first- and secondharmonic signals using twin lock-in amplifiers (Stanford Research Systems 830 ) followed by two digital multimeters and a low-noise current amplifier. ${ }^{6,19,20,22} \mathrm{~A}$ dc bias added to an ac modulation of $6 \mathrm{mV}$ (root-mean-square) at a frequency of $317 \mathrm{~Hz}$ was applied to the sample. The normalized IETS spectra, $\left(\mathrm{d}^{2} / / \mathrm{d} V^{2}\right) /(\mathrm{d} / / \mathrm{d} V)$, were obtained in real time to cancel the effect of junction distance changes while monitoring the signals by the synchronized lock-in amplifiers.

In this work we measured in total 70 and 90 IETS spectra for ODT and ODA, respectively. The continuous IETS measurement under stretching was attempted seven (ODT) and 11 (ODA) times. In four cases for each molecule we were able to assess the continuous stretching of junctions in the lowest conductance range.

Electronic Structure Calculations. To model the geometric and electronic properties of the octane molecular junctions, we use DFT as implemented in the TURBOMOLE software package ${ }^{49}$ with the BP86 generalized gradient functional. ${ }^{50,51}$ As the basis set, we employ def2-SV(P), ${ }^{52}$ which is of split valence quality. Total energies are converged to a precision of better than $10^{-6}$ atomic units, and structure optimizations are carried out until the maximum norm of the Cartesian gradients has fallen below values of $10^{-4}$ atomic units.

Vibrational modes are determined after the geometry optimization by using the "aoforce" module of TURBOMOLE. ${ }^{53} \mathrm{We}$ assume that the "dynamical region" of the molecular junction, i.e., the region where the atoms are allowed to vibrate, is identical to its relaxed part (see Figures $\mathrm{S} 4$ and $\mathrm{S} 5$ of the $\mathrm{Sl}$ ), while we assign an infinite mass to the fixed atoms. We compute vibrational frequencies at every elongation for our optimized model junction structures and characterize the modes by considering the displacement vectors. Due to the larger number of atoms that are free to vibrate and since symmetries are generally absent, the classification of vectors remains approximate only.

Transport Calculations. Using the DFT electronic structure, we determine the elastic transport properties of the molecular contacts in the framework of the Landauer-Büttiker formalism with the help of Green's function techniques. ${ }^{24}$ Our procedure for the computation of IETS spectra, on the other hand, is similar to those described in refs 54 and 55 . We determine the electronvibration coupling within DFT by use of an efficient procedure based on analytic derivatives. The inelastic effects are taken into account in a lowest order expansion of the electric current in terms of the electron-vibration coupling. The details will be described in future work. ${ }^{56}$

Acknowledgment. We thank Juan Carlos Cuevas, Hyunwook Song, Artur Erbe, and Thomas Huhn for stimulating discussions. This work was supported by the Deutsche Forschungsgemeinschaft and by the Baden-Württemberg Stiftung within the Network of Excellence "Functional Nanostructures". F.P. acknowledges support through the Young Investigator Group.

Supporting Information Available: Details on experimental and theoretical procedures. This material is available free of charge via the Internet at http://pubs.acs.org.

\section{REFERENCES AND NOTES}

1. Nitzan, A.; Ratner, M. A. Electron Transport in Molecular Wire Junctions. Science 2003, 300, 1384-1389.

2. Reed, M. A.; Zhou, C.; Muller, C. J.; Burgin, T. P.; Tour, J. M. Conductance of a Molecular Junction. Science 1997, 278, 252-254.

3. Scott, G. D.; Natelson, D. Kondo Resonances in Molecular Devices. ACS Nano 2010, 4, 3560-3579.

4. Venkataraman, L.; Klare, J. E.; Nuckolls, C.; Hybertsen, M. S.; Steigerwald, M. L. Dependence of Single-Molecule Junction Conductance on Molecular Conformation. Nature 2006, 442, 904-907.

5. Yu, L. H.; Zangmeister, C. D.; Kushmerick, J. G. Structural Contributions to Charge Transport across Ni-Octanedithiol Multilayer Junctions. Nano Lett. 2006, 6, 2515-2519.

6. Song, H.; Kim, Y.; Jang, Y. H.; Jeong, H.; Reed, M. A.; Lee, T. Observation of Molecular Orbital Gating. Nature 2009, 462, 1039-1043.

7. Kamenetska, M.; Koentopp, M.; Whalley, A. C.; Park, Y. S.; Steigerwald, M. L.; Nuckolls, C.; Hybertsen, M. S.; Venkataraman, L. Formation and Evolution of Single-Molecule Junctions. Phys. Rev. Lett. 2009, 102, 126803.

8. Li, C.; Pobelov, I.; Wandlowski, T.; Bagrets, A.; Arnold, A.; Evers, F. Charge Transport in Single Au | Alkanedithiol | Au Junctions: Coordination Geometries and Conformational Degrees of Freedom. J. Am. Chem. Soc. 2008, 130, 318326.

9. Ko, C.-H.; Huang, M.-J.; Fu, M.-D.; Chen, C.-H. Superior Contact for Single-Molecule Conductance: Electronic Coupling of Thiolate and Isothiocyanate on $\mathrm{Pt}, \mathrm{Pd}$, and $\mathrm{Au}$. J. Am. Chem. Soc. 2010, 132, 756-764.

10. Paulsson, M.; Krag, C.; Frederiksen, T.; Brandbyge, M. Conductance of Alkanedithiol Single-Molecule Junctions: A Molecular Dynamics Study. Nano Lett. 2009, 9, 117-121.

11. Kiguchi, M.; Tal, O.; Wohlthat, S.; Pauly, F.; Krieger, M.; Djukic, D.; Cuevas, J. C.; van Ruitenbeek, J. M. Highly Conductive Molecular Junctions Based on Direct Binding of Benzene to Platinum Electrodes. Phys. Rev. Lett. 2008, 101, 046801.

12. Venkataraman, L.; Klare, J. E.; Tam, I. W.; Nuckolls, C.; Hybertsen, M. S.; Steigerwald, M. L. Single-Molecule Circuits with Well-Defined Molecular Conductance. Nano Lett. 2006, 6, 458-462.

13. Zotti, L. A.; Kirchner, T.; Cuevas, J.-C.; Pauly, F.; Huhn, T.; Scheer, E.; Erbe, A. Revealing the Role of Anchoring Groups in the Electrical Conduction Through Single-Molecule Junctions. Small 2010, 6, 1529-1535.

14. Chen, F.; Li, X.; Hihath, J.; Huang, Z.; Tao, N. Effect of Anchoring Groups on Single-Molecule Conductance: Comparative Study of Thiol-, Amine-, and CarboxylicAcid-Terminated Molecules. J. Am. Chem. Soc. 2006, 128, 15874-15881.

15. Fagas, G.; Greer, J. C. Tunnelling in Alkanes Anchored to Gold Electrodes via Amine End Groups. Nanotechnology 2007, 18, 424010 . 
16. Zhou, J.; Chen, F.; Xu, B. Fabrication and Electronic Characterization of Single Molecular Junction Devices: A Comprehensive Approach. J. Am. Chem. Soc. 2009, 131, 10439-10446.

17. McDermott, S.; George, C. B.; Fagas, G.; Greer, J. C.; Ratner, M. A. Tunnel Currents across Silane Diamines/Dithiols and Alkane Diamines/Dithiols: A Comparative Computational Study. J. Phys. Chem. C 2009, 113, 744-750.

18. Martin, C. A.; Ding, D.; van der Zant, H. S. J.; van Ruitenbeek, J. M. Lithographic Mechanical Break Junctions for Single-Molecule Measurements in Vacuum: Possibilities and Limitations. New J. Phys. 2008, 10, 065008.

19. Wang, W.; Lee, T.; Kretzschmar, I.; Reed, M. A. Inelastic Electron Tunneling Spectroscopy of an Alkanedithiol SelfAssembled Monolayer. Nano Lett. 2004, 4, 643-646.

20. Kushmerick, J. G.; Lazorcik, J.; Patterson, C. H.; Shashidhar, R.; Seferos, D. S.; Bazan, G. C. Vibronic Contributions to Charge Transport across Molecular Junctions. Nano Lett. 2004, 4, 639-642.

21. Troisi, A.; Ratner, M. A. Molecular Transport Junctions: Propensity Rules for Inelastic Electron Tunneling Spectra. Nano Lett. 2006, 6, 1784-1788.

22. Kim, Y.; Song, H.; Strigl, F.; Pernau, H.-F.; Lee, T.; Scheer, E. Conductance and Vibrational States of Single-Molecule Junctions controlled by Mechanical Stretching and Material Variation. Phys. Rev. Lett. 2011, accepted.

23. González, M. T.; Brunner, J.; Huber, R.; Wu, S.; Schönenberger, C.; Calame, M. Conductance Values of Alkanedithiol Molecular Junctions. New J. Phys. 2008, 10, 065018.

24. Pauly, F.; Viljas, J. K.; Huniar, U.; Häfner, M.; Wohlthat, S.; Bürkle, M.; Cuevas, J. C.; Schön, G. Cluster-based DensityFunctional Approach to Quantum Transport through Molecular and Atomic Contacts. New J. Phys. 2008, 10, 125019.

25. Wohlthat, S.; Pauly, F.; Reimers, J. R. The Conduction Properties of $\alpha, \omega$-Diaminoalkanes and Hydrazine Bridging Gold Electrodes. Chem. Phys. Lett. 2008, 454, 284-288.

26. Fiolhais, F.; Nogueira, F.; Marques, M. A Primer in Density Functional Theory; Springer: Berlin, 2003.

27. Quek, S. Y.; Venkataraman, L.; Choi, H. J.; Louie, S. G.; Hybertsen, M. S.; Neaton, J. B. Amine-Gold Linked SingleMolecule Circuits: Experiment and Theory. Nano Lett. 2007, 7, 3477-3482.

28. Pontes, R. B.; Rocha, A. R.; Sanvito, S.; Fazzio, A.; da Silva, A. J. R. Ab Initio Calculations of Structural Evolution and Conductance of Benzene-1,4-dithiol on Gold Leads. ACS Nano 2011, 5, 795-804.

29. Strange, M.; Rostgaard, C.; Häkkinen, H.; Thygesen, K. S. Self-Consistent GW Calculations of Electronic Transport in Thiol- and Amine- Linked Molecular Junctions. Phys. Rev. B 2011, 83, 115108.

30. Yu, L. H.; Zangmeister, C. D.; Kushmerick, J. G. Origin of Discrepancies in Inelastic Electron Tunneling Spectra on Molecular Junctions. Phys. Rev. Lett. 2007, 98, 206803.

31. Hansma, P. K. Inelastic Electron Tunneling. Phys. Rep. 1977, 30, 145-206.

32. Klein, J.; Léger, A.; Belin, M.; Défourneau, D.; Sangster, M. J. L. Inelastic-Electron-Tunneling Spectroscopy of Metal-Insulator-Metal Junctions. Phys. Rev. B 1973, 7, 23362348.

33. Arroyo, C. R.; Frederiksen, T.; Rubio-Bollger, G.; Vélez, M.; Arnau, A.; Sánchez-Portal, D.; Agraït, N. Characterization of Single-Molecule Pentanedithiol Junctions by Inelastic Electron Tunneling Spectroscopy and First-Principles Calculations. Phys. Rev. B 2010, 81, 075405.

34. Long, D. P.; Lazorcik, J. L.; Mantooth, B. A.; Moore, M. H.; Ratner, M. A.; Troisi, A.; Yao, Y.; Ciszek, J. W.; Tour, J. M.; Shashidhar, R. Effects of Hyfration on Molecular Junction Transport. Nat. Mater. 2006, 5, 901-908.

35. Okabayashi, N.; Paulsson, M.; Ueba, H.; Konda, Y.; Komeda, T. Site Selective Inelastic Electron Tunneling Spectroscopy Probed by Isotope Labeling. Nano Lett. 2010, 10, 2950-2955.

36. Long, D. P.; Troisi, A. Inelastic Electron Tunneling Spectroscopy of Alkane Monolayers with Dissimilar Attachment Chemistry to Gold. J. Am. Chem. Soc. 2007, $129,15303-15310$.
37. Jiang, J.; Kula, M.; Luo, Y. First-Principles Simulations of Inelastic Electron Tunneling Spectroscopy of Molecular Electronic Devices. Nano Lett. 2005, 5, 1551-1555.

38. Solomon, G. C.; Gagliardi, A.; Pecchia, A.; Frauenheim, T.; Carlo, A. D.; Reimers, J. R.; Hush, N. S. Understanding the Inelastic Electron-Tunneling Spectra of Alkanedithiols on Gold. J. Chem. Phys. 2006, 124, 094704.

39. Pecchia, A.; Carlo, A. D.; Gagliardi, A.; Sanna, S.; Frauenheim, T. Incoherent Electron-Phonon Scattering in Octanethiols. Nano Lett. 2004, 4, 2109-2114.

40. Li, Z.; Kosov, D. S. Nature of Well-Defined Conductance of Amine-Anchored Molecular Junctions: Density Functional Calculations. Phys. Rev. B 2007, 76, 035415.

41. Krüger, D.; Fuchs, H.; Rousseau, R.; Marx, D.; Parrinello, M. Pulling Monoatomic Gold Wires with Single Moleules: An Ab Initio Simulation. Phys. Rev. Lett. 2002, 89, 186402.

42. Hihath, J.; Arroyo, C. R.; Rubio-Bollinger, G.; Tao, N.; Agraït, N. Study of Electron-Phonon Interactions in a Single Molecule Covalently Connected to Two Electrodes. Nano Lett. 2008, 8, 1673-1678.

43. Agraït, N.; Untiedt, C.; Rubio-Bollinger, G.; Vieira, S. Onset of Energy Dissipation in Ballistic Atomic Wires. Phys. Rev. Lett. 2002, 88, 216803.

44. Rubio-Bollinger, G.; Bahn, S. R.; Agraït, N.; Jacobsen, K. W.; Vieira, S. Mechanical Properties and Formation Mechanisms of a Wire of Single Gold Atoms. Phys. Rev. Lett. 2001, $87,026101$.

45. van Ruitenbeek, J. M.; Alvarez, A.; Pineyro, I.; Grahmann, C.; Joyez, P.; Devoret, M. H.; Esteve, D.; Urbina, C. Adjustable Nanofabricated Atomic Size Contacts. Rev. Sci. Instrum. 1996, 67, 108.

46. Böhler, T.; Edtbauer, A.; Scheer, E. Point-Contact Spectroscopy on Aluminum Atomic-Size Contacts: Longitudinal and Transverse Vibronic Excitations. New J. Phys. 2009, 11, 013036.

47. Kim, Y.; Song, H.; Kim, D.; Lee, T.; Jeong, H. Noise Characteristics of Charge Tunneling via Localized States in Metal-Molecule-Metal Junctions. ACS Nano 2010, 4, 4426-4430.

48. Champague, A. R.; Pasupathy, A. N.; Ralph, D. C. Mechanically Adjustable and Electrically Gated Single-Molecule Transistors. Nano Lett. 2005, 5, 305-308.

49. Ahlrichs, R.; Bär, M.; Häser, M.; Horn, H.; Kölmel, C. Electronic Structure Calculations on Workstation Computers: The Program System Turbomole. Chem. Phys. Lett. 1989, $162,165-169$.

50. Becke, A. D. Density-Functional Exchange-Energy Approximation with Correct Asymptotic Behavior. Phys. Rev. A 1988, 38, 3098-3100.

51. Perdew, J. P. Density-Functional Approximation for the Correlation Energy of the Inhomogeneous Electron Gas. Phys. Rev. B 1986, 33, 8822-8824.

52. Weigend, F.; Ahlrichs, R. Balanced Basis Sets of Split Valence, Triple Zeta Valence and Quadruple Zeta Valence Quality for H to Rn: Design and Assessment of Accuracy. Phys. Chem. Chem. Phys. 2005, 7, 3297-3305.

53. Deglmann, P.; May, K.; Furche, F.; Ahlrichs, R. Nuclear Second Analytical Derivative Calculations using Auxiliary Basis Set Expansions. Chem. Phys. Lett. 2004, 384, 103107.

54. Viljas, J. K.; Cuevas, J. C.; Pauly, F.; Häfner, M. ElectronVibration Interaction in Transport through Atomic Gold Wires. Phys. Rev. B 2005, 72, 245415.

55. Frederiksen, T.; Paulsson, M.; Brandbyge, M.; Jauho, A.-P. Inelastic Transport Theory from First Principles: Methodology and Application to Nanoscale Devices. Phys. Rev. B 2007, 75, 205413.

56. Bürkle, M.; et al., unpublished. 